\title{
Effect of pulsed laser irradiation on the structure of GeTe films deposited by metal organic chemical vapor deposition: A Raman spectroscopy study
}

\author{
O. Salicio, ${ }^{1, a)}$ C. Wiemer, ${ }^{1}$ M. Fanciulli, ${ }^{1}$ W. Gawelda, ${ }^{2}$ J. Siegel, ${ }^{2}$ C. N. Afonso, ${ }^{2}$ \\ V. Plausinaitiene, ${ }^{3}$ and A. Abrutis ${ }^{3}$ \\ ${ }^{1}$ CNR-INFM MDM Laboratory, via C. Olivetti 2, 20041 Agrate Brianza, Italy \\ ${ }^{2}$ Laser Processing Group, Instituto de Optica, CSIC, Serrano 121, E-28006 Madrid, Spain \\ ${ }^{3}$ Department of General and Inorganic Chemistry, Faculty of Chemistry, Vilnius University, Naugarduko 24, \\ LT-2006 VU, Lithuania
}

(Received 23 October 2008; accepted 20 December 2008; published online 11 February 2009)

\begin{abstract}
Phase changes between amorphous and crystallized states were induced by laser irradiation with nanosecond pulses in $\mathrm{Ge}_{x} \mathrm{Te}_{y}$ films grown by metal organic chemical vapor deposition. The different phases were obtained by adjusting the pulse energy and could be distinguished by their different optical reflectivities. The corresponding structural changes were studied by Raman spectroscopy, showing marked differences for the two phases. A clear correlation is found between optical reflectivity levels, crystallographic state and the evolution of $\mathrm{Ge}-\mathrm{Ge}, \mathrm{Te}-\mathrm{Te}$, and $\mathrm{Ge}-\mathrm{Te}$ Raman bands. (C) 2009 American Institute of Physics. [DOI: 10.1063/1.3075906]
\end{abstract}

\section{INTRODUCTION}

Chalcogenide materials based on $\mathrm{Ge}, \mathrm{Sb}$, and Te have been implemented in optical storage technology for decades and are now under evaluation for the next generation of nonvolatile memory devices. ${ }^{1,2}$ Actually, the fast phase change between amorphous and crystallized states of these materials, which is associated with the variation of the electrical resistivity by several orders of magnitude, ${ }^{3}$ can be induced by ultrafast optical or electrical pulses. So far, these materials have been deposited by rf-magnetron sputtering, an anisotropic deposition technique, which is not conformal enough for fabricating high aspect ratio structures for next generation of phase change memory devices. Metal organic chemical vapor deposition (MOCVD) is under investigation as a promising route for obtaining such structures. ${ }^{4-11}$ Thus, the understanding of the structural evolution at the microscale upon phase change is a key point to optimize memories based on phase change materials.

Among several experimental techniques that can follow the variation of the electronic structure and chemical bonding taking place during the phase transition, namely $\mathrm{x}$-ray photoelectron spectroscopy (XPS), ${ }^{12}$ spectroscopic ellipsometry, ${ }^{13}$ and extended $\mathrm{x}$-ray absorption fine structure (EXAFS), ${ }^{14,15}$ micro-Raman spectroscopy has the advantage of being a local technique that can, in principle, capture the vibration of interatomic bonds occurring in the course of the phase transition. This technique has been recently applied to the study of chalcogenide materials, ${ }^{16,17}$ including the in situ monitoring of phase transitions in Ge-Te-Si alloys. ${ }^{18}$

In this paper, we study the structural properties of $\mathrm{Ge}_{x} \mathrm{Te}_{y}$ films grown by MOCVD on $\mathrm{Si} / \mathrm{SiO}_{2}$ substrates upon laser-induced phase change. We demonstrate phase change

\footnotetext{
${ }^{a)}$ Author to whom correspondence should be addressed. Electronic mail: olivier.salicio@mdm.infm.it.
}

by pulsed laser irradiation and we follow the induced structural changes by Raman spectroscopy, relating them to different reflectivity levels.

\section{EXPERIMENTAL}

$\mathrm{Ge}_{0.79} \mathrm{Te}_{1.00}$ thin films (30 nm) have been grown on $\mathrm{SiO}_{2}$ $(40 \mathrm{~nm}) / \mathrm{Si}(100)$ substrates by MOCVD using pulsed liquid injection/flash evaporation principle of precursor microdoses. ${ }^{19} \mathrm{Ge}\left(\mathrm{NMe}_{2}\right)_{4}$ and $\mathrm{Te}\left({ }^{i} \mathrm{Pr}\right)_{2}$ solutions in toluene were used as precursors and nitrogen as a carrier gas.

Pulsed laser irradiation was performed on the as-grown $\mathrm{Ge}_{x} \mathrm{Te}_{y}$ films in order to induce local phase changes. The laser used was a $Q$-switched Ti:sapphire laser operating at $800 \mathrm{~nm}$ wavelength with a pulse duration of $8 \mathrm{~ns}$. The $s$-polarized Gaussian beam was focused onto the sample at an angle of incidence of $54^{\circ}$ to an elliptical spot size of $100 \times 59 \mu \mathrm{m}^{2}\left(1 / e^{2}\right.$ intensity diameter $){ }^{20}$ The induced phase change was observed in situ with an optical microscope composed of a long working distance objective lens (numerical aperture, NA=0.42) and a tube lens in a configuration along the normal to the sample surface. Illumination was provided by a light emitting diode and the magnified images $(20 \times)$ were recorded by a 12-bit charge coupled device camera. A quantitative analysis of the reflectivity change produced by laser irradiation was obtained by dividing the recorded images of the laser exposed region by a reference image of the unexposed sample and extracting horizontal cross sections through the center of the resulting images. The so-obtained reflectivity profiles are normalized to the reflectivity of the unexposed film.

Raman measurements were carried out with a Renishaw 2000 spectrometer using a $633 \mathrm{~nm}$ laser excitation source on both the as-grown film and the irradiated regions. In order to minimize the thermal effects of long-time laser exposure, we used a low-power excitation with a $50 \times$ magnification objective $(\mathrm{NA}=0.75)$. In this configuration, the excitation 


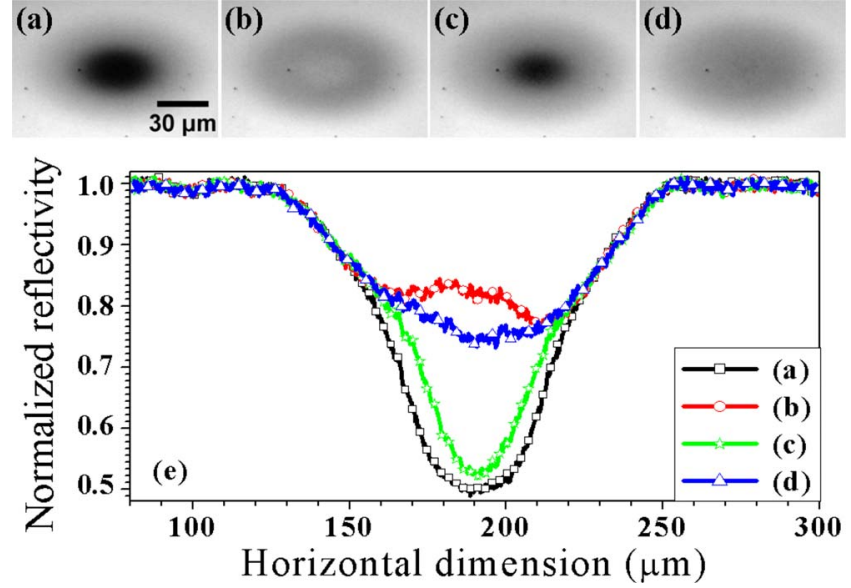

FIG. 1. (Color online) A sequence of in situ optical micrographs of the laser-irradiated surface of a $\mathrm{Ge}_{x} \mathrm{Te}_{y}$ thin film deposited by MOCVD. (a) Laser-induced amorphization (low-reflectivity state) upon intense single pulse irradiation (SP1). (b) Partial recovery of the initial crystallinity (highreflectivity state) upon multiple pulse irradiations (MP1). [(c) and (d)] Subsequent phase cycle between both states, amorphization [SP2, (c)] and recrystallization [MP2 (d)]. (e) Horizontal reflectivity profiles through the center of the spots shown in (a)-(d), normalized to the reflectivity of the as-grown film.

power density on the sample was on the order of $0.3 \mathrm{~W} / \mathrm{mm}^{2}$. The Raman spectra presented correspond to an average of ten spectra with an exposure time of $60 \mathrm{~s}$ for each spectrum.

Subsequent annealing of the as-grown sample was carried out in a furnace under $\mathrm{N}_{2}$ flux. X-ray reflectometry (XRR), total reflection X-ray fluorescence (TXRF), and X-ray diffraction (XRD) were also performed on the as-grown and annealed samples in order to investigate the crystallographic structure, the layer structure, and the chemical composition. XRR and TXRF were performed using an X-ray reflectometer total reflection spectrometer equipped with Mo $K \alpha$ radiation source. XRD was performed on an X-ray diffractometer equipped with $\mathrm{Cu} K \alpha$ radiation source, a four circle goniometer and a curved position-sensitive detector.

\section{RESULTS}

The laser irradiation protocol consisted of first performing a sequence of single pulse irradiations at increasing pulse energies on unexposed regions of the sample, which allowed to determine the threshold laser fluences for phase change $\left(\approx 50 \mathrm{~mJ} / \mathrm{cm}^{2}\right)$ and ablation $\left(\approx 85 \mathrm{~mJ} / \mathrm{cm}^{2}\right)$ in the as-grown film. Phase change was accompanied by a marked decrease of reflectivity, consistent with local amorphization of a crystalline film. Then, a series of single pulse (SP) irradiations, at constant energy $\left(74 \mathrm{~mJ} / \mathrm{cm}^{2}\right)$, on unexposed regions was performed, leading to a series of relatively large amorphized regions. An example of such an amorphized region (SP1), characterized by a lower reflectivity than the as-grown film, is shown in Fig. 1(a). Recrystallization using single laser pulses was not possible, which is consistent with the minimum pulse duration reported for crystallization of amorphous GeTe being $30 \mathrm{~ns}^{21}$ Therefore, multiple pulse (MP) irradiations (100 pulses) at increasing pulse energies on these amorphous regions were performed in order to identify the
TABLE I. Irradiation procedures followed in order to obtain the spots with different reflectivity levels: SP and MP at the specified laser fluences.

\begin{tabular}{lc}
\hline \hline Spot number & Irradiation procedure, laser fluence used \\
\hline SP1 & Single pulse on as-grown film, $74 \mathrm{~mJ} \mathrm{~cm}^{-2}$ \\
MP1 & 100 pulses on spot SP1, $28 \mathrm{~mJ} \mathrm{~cm}^{-2}$ \\
SP2 & Single pulse on MP1, 74 mJ cm \\
MP2 & 100 pulses on spot SP2, $28 \mathrm{~mJ} \mathrm{~cm}^{-2}$ \\
\hline \hline
\end{tabular}

optimum laser fluence for recrystallization $\left(28 \mathrm{~mJ} / \mathrm{cm}^{2}\right)$. An example of a so-recrystallized region after one complete phase cycle (SP1+MP1) is shown in Fig. 1(b). Further irradiations of these recrystallized regions, aimed at inducing reamorphization, were performed at laser fluences slightly lower than the fluence chosen to induce the first amorphization. In this way the slightly higher absorption of the laser recrystallized regions compared to as-grown regions [visibly lower reflectivity for instance in Fig. 1(b)] was taken into account. The so-obtained reamorphized regions [Fig. 1(c), spot SP2] showed a similar optical contrast as the amorphized regions directly from the as-grown film [Fig. 1(a)]. Consecutive irradiation using multiple laser pulses at $28 \mathrm{~mJ} / \mathrm{cm}^{2}$ induced again recrystallization [Fig. 1(d), spot MP2, two phase cycles] with a similar optical contrast as the region obtained after one phase cycle [Fig. 1(b)]. The irradiation procedures and laser fluences are summarized in Table I.

The induced reflectivity changes have been quantified and are shown in form of horizontal cross sections through the spot center in Fig. 1(e). The maximum reflectivity change obtained upon amorphization in the first phase cycle is remarkably high, approximately $-50 \%[R \approx 0.5$, minimum of curve (a)]. The partial recovery of the reflectivity level upon multiple pulse irradiations at low fluence is $R \approx 0.8$ [curve (b)]. The relative optical contrast (OC) between both states is thus $\mathrm{OC} \approx 0.3$. The reflectivity profiles remain similar in the second cycle [curves (c) and (d)], although the OC becomes slightly smaller.

Raman analysis was performed in the very center of these selected spots that are representative for the different states of laser-induced phase changes.

Figure 2 compares the Raman spectrum of the as-grown sample to those of spots MP1, MP2, SP1, and SP2. Four main contributions, with various intensities, are clearly visible in these spectra at $162,218,275$, and $300 \mathrm{~cm}^{-1}$. These bands are in a good agreement with the Raman bands previously reported in literature for GeTe compounds. Andrikopoulos et al. ${ }^{16,17}$ described four main contributions for GeTe thin films: (i) a high intensity band centered at $\sim 162 \mathrm{~cm}^{-1}$, attributed to Ge-Te bonds in amorphous GeTe; ${ }^{16,17}$ (ii) a band at $\sim 275 \mathrm{~cm}^{-1}$, attributed to amorphous $\mathrm{Ge}-\mathrm{Ge}$ bonds; ${ }^{16}$ (iii) a band at $\sim 300 \mathrm{~cm}^{-1}$ corresponding to $\mathrm{Ge}-\mathrm{Ge}$ bonds in crystallized GeTe (also observed by Kolobov et al. ${ }^{14}$ for crystallized GeTe thick films) and (iv) a large band around $218 \mathrm{~cm}^{-1}$ already observed only for amorphous GeTe. ${ }^{16}$ An additional band, corresponding to amorphous GeTe, has been reported at $182 \mathrm{~cm}^{-1} \cdot{ }^{16,17}$ Moreover, Andrikopoulos et al. ${ }^{16,17}$ and Kolobov et al. observed four 

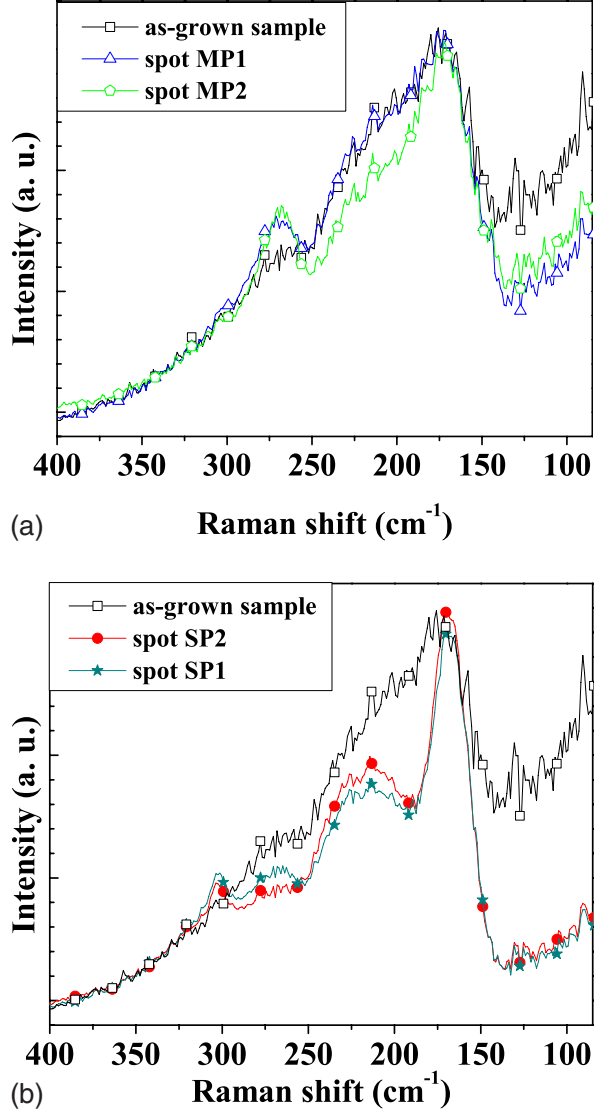

FIG. 2. (Color online) (a) Comparison of the Raman spectra of the as-grown sample and spots MP1 and MP2. (b) Comparison of the Raman spectra of spots SP1 and SP2 and of the as-grown matrix.

bands in the $150-50 \mathrm{~cm}^{-1}$ range: at $\sim 145, \sim 127, \sim 111$, and $\sim 88 \mathrm{~cm}^{-1}$. All these observations are summarized in Table II.

In literature, Raman peak positions of single elements are referred as follow: $\mathrm{Ge}-\mathrm{Ge}, 282.5-300 \mathrm{~cm}^{-1}$ range for Ge nanocrystals ${ }^{22,23} \mathrm{Te}-\mathrm{Te}, \sim 141 \mathrm{~cm}^{-1}$ for crystal of Te (Ref. 24) and $\mathrm{Sb}-\mathrm{Sb}, 150-155 \mathrm{~cm}^{-1}$ range for amorphous $\mathrm{Sb}^{25}$

\section{DISCUSSION}

According to the Raman data summarized in Table II, the band observed in Fig. 2 at $\sim 162 \mathrm{~cm}^{-1}$ can be attributed to an overlap between $\mathrm{Ge}-\mathrm{Te}$ and $\mathrm{Te}-\mathrm{Te}$ bonds and those at $\sim 218,275$, and $300 \mathrm{~cm}^{-1}$ to Ge-Ge bonds. The spectrum of the as-grown sample displays a broad band from 150 to $250 \mathrm{~cm}^{-1}$ and a second band centered on $\sim 275 \mathrm{~cm}^{-1}$. The origin of the broad band is consistent with an overlap of contributions: the Ge-Te and Te-Te overlap observed around $162 \mathrm{~cm}^{-1}$ (Refs. 16 and 17) and a second contribution around $218 \mathrm{~cm}^{-1}$. ${ }^{16,17}$ The band around $275 \mathrm{~cm}^{-1}$ is consistent with the signal from $\mathrm{Ge}-\mathrm{Ge}$ bonds reported for amorphous GeTe. ${ }^{16,17}$

As shown on Figs. 2(a) and 2(b), Raman spectra could be divided into two groups: (i) spectra of spots MP1 and MP2 are similar to those of the as-grown film [Fig. 2(a)], supporting further that both spots are in a similar crystallographic state as the as-grown sample; (ii) spectra of spots SP1 and SP2, which are significantly different from the one of the as-grown film [Fig. 2(b)].

The Raman spectra of the as-grown material, MP1 and SP1 spots and the corresponding deconvolution done by using the band position reported in literature (Table II) are presented in Fig. 3. Fitting parameters are summarized in Table III. The same deconvolution procedure was performed on MP1 and SP1 spots, as shown on Figs. 3(b) and 3(c), respectively. In the spectral fit, Andrikopoulos et al. ${ }^{16,17}$ used seven contributions in the $250-80 \mathrm{~cm}^{-1}$ range. In our case, at least four contributions must be set to complete a reasonable fitting: $\sim 218, \sim 182, \sim 162$, and $\sim 88 \mathrm{~cm}^{-1}$. The low intensity of the other contributions in the $150-100 \mathrm{~cm}^{-1}$ range (i.e., $\sim 145, \sim 127$, and $\sim 111 \mathrm{~cm}^{-1}$ ) leads us not to consider them as significant parameter for the fitting. The positions of the Gaussian curves used to fit the as-grown spectrum [Fig. 3(a)] are in good agreement with those reported by Andrikopoulos et al. ${ }^{16}$ in the $250-80 \mathrm{~cm}^{-1}$ range. To fit properly the $350-250 \mathrm{~cm}^{-1}$ range, two additional Gaussian curves around 300 (Ref. 14) and $275 \mathrm{~cm}^{-1}$ (Ref. 17) corresponding both to Ge-Ge bonds were used. A good fitting was obtained after the adjustment of the full width at half maximum (FWHM) of each Gaussian contribution.

By comparing the as-grown, MP1 and MP2 spectra, i.e., Fig. 2(a), it can be seen that the signal at $275 \mathrm{~cm}^{-1}$ is higher for MP1 and MP2 than for the as-grown film. In this wavenumber range, the $\mathrm{Ge}-\mathrm{Ge}$ band measured in amorphous GeTe is present. ${ }^{17}$ Therefore, the "recrystallized" regions in MP1 and MP2 have a higher number of Ge-Te bonds than

TABLE II. Nonexhaustive Raman shift response of GeTe reported in literature ( $a$-GeTe and $c$-GeTe refer to amorphous and crystalline GeTe, respectively).

\begin{tabular}{|c|c|c|c|}
\hline $\begin{array}{l}\text { Raman shift } \\
\left(\mathrm{cm}^{-1}\right)\end{array}$ & Corresponding bonds & Compounds & Reference \\
\hline$\sim 88$ & $\mathrm{Ge}-\mathrm{Te}$ & $a$-GeTe and $c$-GeTe & 14,16 , and 17 \\
\hline $111-127$ & $\mathrm{Ge}-\mathrm{Te}$ & $a$-GeTe and $c$-GeTe & 14,16 , and 17 \\
\hline $155-162$ & $\mathrm{Ge}-\mathrm{Te}+\mathrm{Te}-\mathrm{Te}$ overlap & $a-\mathrm{GeTe}$ & 16 and 17 \\
\hline 182 & $\mathrm{C} 2$ edge sharing & $a-\mathrm{GeTe}$ & 16 and 17 \\
\hline 218 & & $a-\mathrm{GeTe}$ & 16 and 17 \\
\hline 275 & $\mathrm{Ge}-\mathrm{Ge}$ & $\begin{array}{l}a-\mathrm{GeTe} \text { (attributed to cluster size } 1 \\
\mathrm{~nm} \text { by Andrikopoulos } \text { et } a l^{\mathrm{a}}{ }^{\mathrm{a}} \text { ) }\end{array}$ & 17 \\
\hline$\sim 300$ & $\mathrm{Ge}-\mathrm{Ge}$ & $c-\mathrm{Ge}-\mathrm{Ge}$ and $c-\mathrm{Ge}-\mathrm{Te}$ & 14 \\
\hline
\end{tabular}

${ }^{\mathrm{a}}$ Reference 17. 

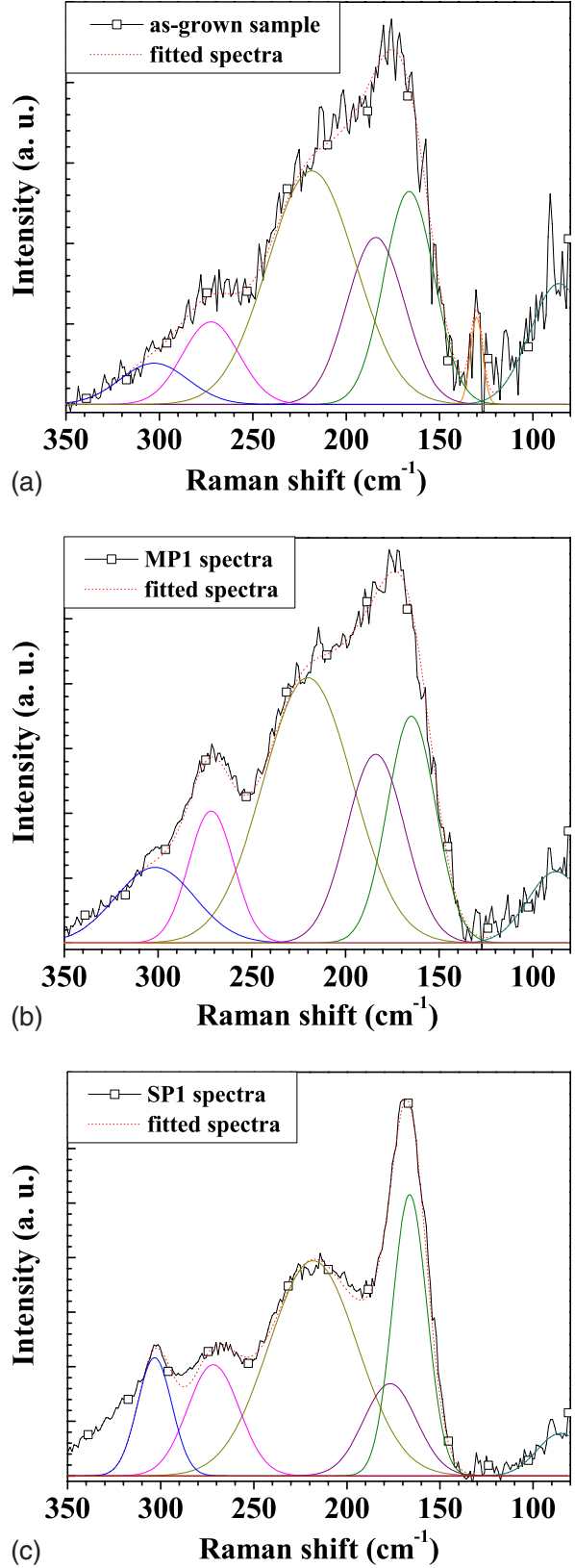

FIG. 3. (Color online) Spectral deconvolution of the as-grown (a), MP1 (b) and SP1 (c) Raman spectra using the fitting parameters summarized in Table III.

found in the as-grown film, corresponding to a less crystallized state. This is consistent with the optical reflectivity measurements shown in Fig. 1(e). The recrystallized region has a reflectivity that is $\approx 20 \%$ lower than the as-grown film, which can be explained by its lower degree of crystallization as compared to the as-grown material. Indeed, no band was observed by Andrikopoulos et al. ${ }^{17}$ in this range for crystallized GeTe. From this result, we could conclude that the as-grown GeTe sample is only partially crystallized.

The Raman response of SP1 and SP2 shows a very similar strong response in the $300-140 \mathrm{~cm}^{-1}$ range [Fig. 2(b)], suggesting that both the amorphization following recrystallization (SP2) and amorphization of the as-grown films (SP1) lead to very similar $\mathrm{Ge}-\mathrm{Ge}$ and $\mathrm{Ge}-\mathrm{Te}$ bond strengths, consistently with the comparable reflectivity levels shown on
Fig. 1(e). Significant differences are instead observed when comparing the Raman response of SP1 and SP2 to that of the as-grown material, that become easier to be visualized by comparing the deconvolution performed on SP1 spectra [Fig. $3(\mathrm{c})]$ to that of the as-grown film [Fig. 3(a)[. The two peaks around 182 and $162 \mathrm{~cm}^{-1}$ are only present for amorphous GeTe according to Andrikopoulos et al. ${ }^{16,17}$ and assigned to the edge-sharing tetrahedra $\mathrm{GeTe}_{4}$.

Moreover, the intensity of the bands around 275 and $300 \mathrm{~cm}^{-1}$, which are both assigned to $\mathrm{Ge}-\mathrm{Ge}$ bonds, strongly increases as compared to the signal of the as-grown film. This behavior could have different origins: on one hand it could be attributed to the evolution of the crystallographic state or the amorphization leading to a reordering of the material bonds. On the other hand, the process could be interpreted as desorption of Te from the material upon both single and multiple laser pulse irradiations. Given that Te species are known to be more volatile than Ge atoms, the desorption of Te species would favor the observation of stronger signals related to $\mathrm{Ge}-\mathrm{Ge}$ bonds. Moreover, this interpretation is consistent with (i) the decrease in the 218 and $170 \mathrm{~cm}^{-1}$ peaks, attributed to Te-related bonds in amorphous Ge-Te; (ii) the increase in the 300 and $275 \mathrm{~cm}^{-1}$ peaks, assigned to $\mathrm{Ge}-\mathrm{Ge}$ bonds in crystallized $\mathrm{Ge}$ and $\mathrm{GeTe}$ and to amorphous $\mathrm{Ge}-\mathrm{Ge}$ bonds in GeTe, respectively, (iii) the possible presence of an additional band at $140 \mathrm{~cm}^{-1}$ in the spectrum of the annealed sample, assigned to Te-Te bonds. ${ }^{18,24}$

A complementary XRD analysis of the as-grown sample was performed to further assert the crystallographic nature of the as-grown film. No observable diffraction peaks were detected (data not shown here), most likely due to the small grain size. In order to further investigate whether the asgrown sample is only partially crystallized, annealing at $280{ }^{\circ} \mathrm{C}$ under $\mathrm{N}_{2}$ atmosphere was performed to induce its full crystallization. Figure 4(a) compares the Raman spectra of the as-grown sample and the annealed one. Upon annealing, an increase of the 275 and $300 \mathrm{~cm}^{-1}$ peaks and a decrease of the 218,182 , and $162 \mathrm{~cm}^{-1}$ peaks are observed. These observations are confirmed by the deconvolution of the Raman spectra of this sample as summarized in Fig. 4(b). The interpretation of this behavior is not trivial, since not only crystallographic changes but also chemical variations are caused by the annealing process. Indeed, XRD performed on the annealed sample reveals weakly pronounced peaks belonging to the Te phase, ${ }^{26}$ as shown in Fig. 5. TXRF measurements performed on the as-grown sample reveal a $\mathrm{Ge}_{0.79} \mathrm{Te}_{1.00}$ film composition (Fig. 6). Both the Te and $\mathrm{Ge}$ related signals decrease upon annealing. The area corresponding to Te decreases by $51 \%$, whereas the one corresponding to Ge by $74 \%$. Both Ge and Te desorptions, probably also due to the evaporation of the corresponding oxides, might have taken place during the annealing process. The chemical composition after annealing was determined to be $\mathrm{Ge}_{0.44} \mathrm{Te}_{1.00}$ (Fig. 6). The compositional change and the changes in the diffraction pattern upon annealing are consistent with $\mathrm{Ge}$ and Te desorption, followed by phase segregation and crystallization of $\mathrm{Te}$, indicating the formation of $\mathrm{Te}-\mathrm{Te}$ bonds in the film. These results reveal the variation of the bond strength not only upon thermal annealing but also 
TABLE III. Peak parameters fitting: Deconvolution peak positions and Gaussian FWHM and maximum peak intensities normalized to the highest peak.

\begin{tabular}{|c|c|c|c|c|c|c|c|}
\hline \multicolumn{2}{|c|}{ As-grown sample } & \multicolumn{2}{|c|}{ MP1 spot } & \multicolumn{2}{|c|}{ SP1 spot } & \multicolumn{2}{|c|}{$280{ }^{\circ} \mathrm{C}$ annealed sample } \\
\hline $\begin{array}{l}\text { Peak position } \\
\qquad(\mathrm{FWHM}) \\
\left(\mathrm{cm}^{-1}\right)\end{array}$ & $\begin{array}{c}\text { Peak } \\
\text { intensity }\end{array}$ & $\begin{array}{l}\text { Peak position } \\
(\mathrm{FWHM}) \\
\left(\mathrm{cm}^{-1}\right)\end{array}$ & $\begin{array}{c}\text { Peak } \\
\text { intensity }\end{array}$ & $\begin{array}{l}\text { Peak position } \\
(\mathrm{FWHM}) \\
\left(\mathrm{cm}^{-1}\right)\end{array}$ & $\begin{array}{c}\text { Peak } \\
\text { intensity }\end{array}$ & $\begin{array}{l}\text { Peak position } \\
\text { (FWHM) }\end{array}$ & $\begin{array}{c}\text { Peak } \\
\text { intensity }\end{array}$ \\
\hline 86.1 & & 87.7 & & 85.4 & & 87.8 & \\
\hline$(36.4)$ & 42 & $(35.3)$ & 24 & (29.4) & 15 & $(35.7)$ & 43 \\
\hline 130.2 & & & & & & 123.6 & \\
\hline \multirow[t]{2}{*}{ (7.7) } & 30 & $\cdots$ & $\cdots$ & $\cdots$ & $\cdots$ & $(16.1)$ & 18 \\
\hline & & & & & & 144.6 & \\
\hline$\cdots$ & $\cdots$ & $\cdots$ & $\cdots$ & $\cdots$ & $\cdots$ & $(4.2)$ & 40 \\
\hline 166.3 & & 164.8 & & 166.3 & & 166.3 & \\
\hline$(31.5)$ & 74 & $(30.3)$ & 77 & (21.7) & 97 & $(23.1)$ & 35 \\
\hline 184.2 & & 183.8 & & 176.8 & & 184.2 & \\
\hline$(36.4)$ & 58 & $(36.6)$ & 64 & (33.4) & 32 & $(31.5)$ & 41 \\
\hline 218.5 & & 220.1 & & 218.1 & & 222.3 & \\
\hline$(55.3)$ & 82 & $(56.3)$ & 91 & $(56.1)$ & 74 & (49.7) & 42 \\
\hline 272.4 & & 271.7 & & 271.7 & & 272.8 & \\
\hline$(35.8)$ & 29 & $(28.2)$ & 45 & $(32.7)$ & 38 & (25.9) & 100 \\
\hline 302.9 & & 301.5 & & 303.2 & & 302.5 & \\
\hline$(42.8)$ & 14 & $(49.7)$ & 26 & (21.7) & 41 & $(18.2)$ & 67 \\
\hline
\end{tabular}

upon laser irradiation. Therefore, phase change seems to promote phase segregation upon recrystallization, with an increase in $\mathrm{Ge}-\mathrm{Ge}$ related bonds and a reduction of $\mathrm{Ge}-\mathrm{Te}$ related ones, as can be concluded by comparing the spectra of the as-grown matrix with the one of the MP2 spot.

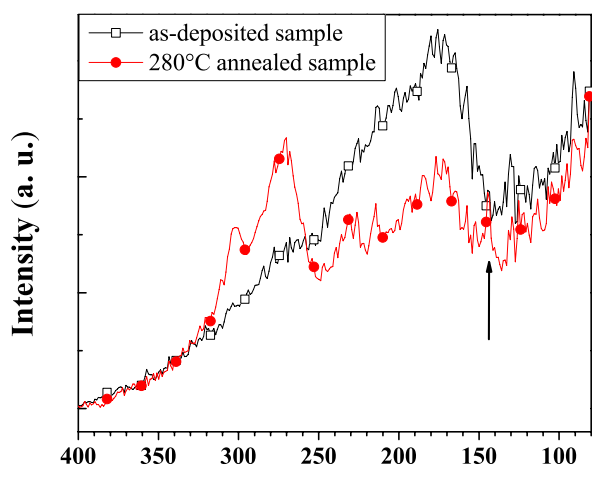

(a)
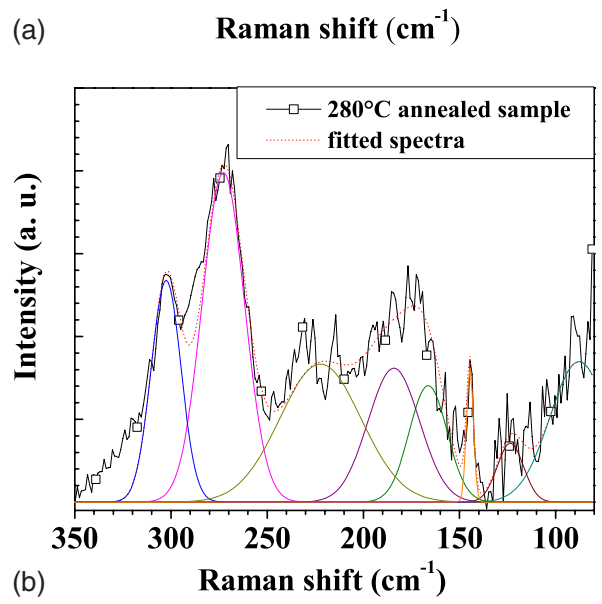

FIG. 4. (Color online) (a) Raman spectra of the as-deposited and $280{ }^{\circ} \mathrm{C}$ annealed GeTe sample. The position where the Te-Te related band develops is indicated by an arrow. (b) Deconvolution of the Raman spectra of the annealed sample according to the fitting parameters reported in Table III

\section{CONCLUSION}

Phase changes between amorphous and crystallized states have been induced using ns laser pulses in $\mathrm{Ge}_{0.79} \mathrm{Te}_{1.00}$ films grown by MOCVD. Phase change takes place between a fully amorphized state induced by single pulses at $74 \mathrm{~mJ} / \mathrm{cm}^{2}$ and a partially crystallized state obtained by multiple pulse irradiation at $28 \mathrm{~mJ} / \mathrm{cm}^{2}$. Although multiple phase cycling was demonstrated on the same spot, a variation of the crystallized state, corresponding to different levels of optical reflectivity and differences in the Raman spectra, is revealed already after the first cycle. It is observed that the Raman band at $275 \mathrm{~cm}^{-1}$ develops upon repetitive phase change when recrystallization is induced on previously amorphized spots and that this band is still present after further amorphization, corresponding to an increase of $\mathrm{Ge}-\mathrm{Ge}$ bonds after cycling on the same spot. Moreover, a weak band at $300 \mathrm{~cm}^{-1}$ present in Raman spectra of amorphized regions is hardly appreciable in spectra of recrystallized regions and is further reduced in the spectrum of the as-grown film. Desorption of $\mathrm{Ge}$ and $\mathrm{Te}$, together with phase separation and $\mathrm{Ge}$

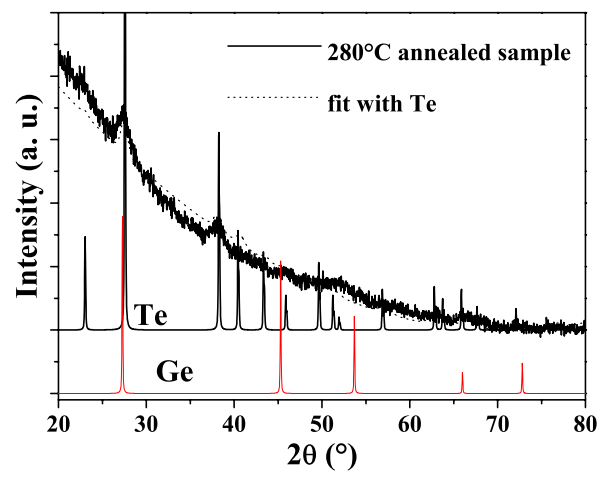

FIG. 5. (Color online) XRD data and corresponding simulation with Te phase. The diffraction patterns reported for Te and Ge powders (Ref. 26) are also added for comparison. 


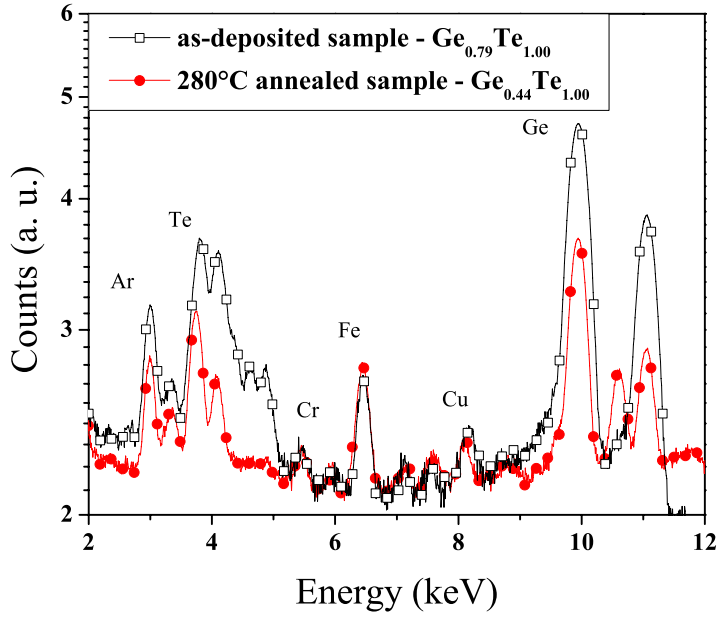

FIG. 6. (Color online) TXRF spectrum of the $30 \mathrm{~nm}$ thick $\mathrm{Ge}_{x} \mathrm{Te}_{y}$ film before and after annealing.

segregation, as indicated by Raman spectroscopy supported by XRD and TXRF measurements, are suggested to be responsible for the lower optical contrast achieved during the second phase cycle.

\section{ACKNOWLEDGMENTS}

This work was partially supported by the European Project CHEMAPH, FP6, under Grant No. N 027561.

${ }^{1}$ S. Hudgens and B. Johnson, MRS Bull.. 29, 829 (2004).

${ }^{2}$ A. L. Lacaita, D. Ielmini, and D. Mantegazza, Solid-State Electron. 52, 1443 (2008).

${ }^{3}$ A. L. Lacaita, Solid-State Electron. 50, 24 (2006).

${ }^{4}$ S. R. Ovshinsky and S. Kamepalli, U.S. Patent No. PCT/US2006/000382, (2006).

${ }^{5}$ R.-Y. Kim, H.-G. Kim, and S.-G. Yoon, Appl. Phys. Lett. 89, 102107 (2006).

${ }^{6}$ B. J. Choi, S. Choi, Y. C. Shin, C. S. Hwang, J. W. Lee, J. Jeong, Y. J. Kim, S.-Y. Hwang, and S. K. Hongc, J. Electrochem. Soc. 154, H318 (2007).
${ }^{7}$ B. J. Choi, S. Choi, Y. C. Shin, K. M. Kim, C. S. Hwang, Y. J. Kim, Y. J. Son, and S. K. Hong, Chem. Mater. 19, 4387 (2007).

${ }^{8}$ G. S. Tompa, S. Sun, C. E. Rice, J. Cuchiaro, and E. Dons, Mater. Res. Soc. Symp. Proc. 997, I10 (2007).

${ }^{9}$ A. Abrutis, V. Plausinaitiene, M. Skapas, C. Wiemer, O. Salicio, A. Pirovano, E. Varesi, S. Rushworth, W. Gawelda and J. Siegel, Chem. Mater. 20, 3557 (2008)

${ }^{10}$ R.-Y. Kim, H.-G. Kim, and S.-G. Yoon, J. Electrochem. Soc. 155, D137 (2008).

${ }^{11}$ M. Longo, O. Salicio, C. Wiemer, R. Fallica, A. Molle, M. Fanciulli, C. Giesen, B. Seitzinger, P. K. Baumann, M. Heuken, and S. Rushworth, Growth study of $\mathrm{Ge}_{x} \mathrm{Sb}_{y} \mathrm{Te}_{z}$ deposited by MOCVD under nitrogen for non volatile memory applications, J. Cryst. Growth 310, 5035 (2008)

${ }^{12}$ L. Bo, S. Zhi-Tang, Z. Ting, F. Song-Lin, and C. Bomy, Chin. Phys. 13, 1947 (2004).

${ }^{13}$ J. W. Park, S. H. Baek, T. D. Kang, H. Lee, Y. S. Kang, T. Y. Lee, D. S. Suh, K. J. Kim, C. K. Kim, Y. H. Khang, J. L. F. Da Silva, and S. H. Wei, J. Appl. Phys. 93, 021914 (2008).

${ }^{14}$ A. V. Kolobov, P. Fons, J. Tominaga, A. L. Ankudinov, S. N. Yannopoulos, and K. S. Andrikopoulos, J. Phys.: Condens. Matter 16, S5103 (2004).

${ }^{15}$ Y. Kim, M. H. Jang, K. Jeong, M. H. Cho, K. H. Do, D. H. Ko, H. C. Sohn, and M. G. Kim, Appl. Phys. Lett. 92, 061910 (2008).

${ }^{16}$ K. S. Andrikopoulos, S. N. Yannopoulos, G. A. Voyiatzis, A. V. Kolobov, M. Ribes, and J. Tominaga, J. Phys.: Condens. Matter 18, 965 (2006).

${ }^{17}$ K. S. Andrikopoulos, S. N. Yannopoulos, A. V. Kolobov, P. Fons, and J. Tominaga, J. Phys. Chem. Solids 68, 1074 (2007).

${ }^{18}$ M. Anbarasu, S. Asokan, S. Prusty, and A. K. Sood, Appl. Phys. Lett. 91, 093520 (2007)

${ }^{19}$ J. P. Sénateur, C. Dubourdieu, V. Galindo, F. Weiss, and A. Abrutis, in Innovative Processing of Films and Nanocrystalline Powders, edited by K.-L. Choy (Imperial College Press, London, 2002), p. 71.

${ }^{20}$ J. Siegel, W. Gawelda, D. Puerto, C. Dorronsoro, J. Solis, C. N. Afonso, J. C. G. De Sande, R. Bez, A. Pirovano, and C. Wiemer, J. Appl. Phys. 103, 023516 (2008).

${ }^{21}$ M. Chen, K. A. Rubin, and R. W. Barton, Appl. Phys. Lett. 49, 502 (1986).

${ }^{22}$ W. K. Choi, V. Ng, S. P. Ng, H. H. Thio, Z. X. Shen, and W. S. Li, J. Appl. Phys. 86, 1398 (1999).

${ }^{23}$ Z. F. Krasil'nik and P. Lytvyn, D. N. Lobanov, N. Mestres, A. V. Novikov, J. Pascual, M. Y. Valakh, and V. A. Yukhymchuk, Nanotechnology 13, 81 (2002).

${ }^{24}$ A. S. Pine and G. Dresselhaus, Phys. Rev. B 4, 356 (1971).

${ }^{25}$ J. S. Lannin, Phys. Rev. B 15, 3863 (1977).

${ }^{26}$ Inorganic Crystal Structure Database fine No. 65692 for Te and No. 44610 for Ge, Fachinformationszentrum Karlsruhe, 2008. 